\title{
Acupuncture Therapy Is More Effective Than Artificial Tears for Dry Eye Syndrome: Evidence Based on a Meta-Analysis
}

\author{
Lei Yang, ${ }^{1}$ Zongguo Yang, ${ }^{2}$ Hong Yu, ${ }^{1}$ and Hui Song ${ }^{1}$ \\ ${ }^{1}$ Aerospace Center Hospital, Beijing 100049, China \\ ${ }^{2}$ Shanghai Public Health Clinical Center, Fudan University, Shanghai 201508, China \\ Correspondence should be addressed to Hui Song; songhui731@sina.com
}

Received 14 November 2014; Revised 26 February 2015; Accepted 20 March 2015

Academic Editor: Evan P. Cherniack

Copyright (c) 2015 Lei Yang et al. This is an open access article distributed under the Creative Commons Attribution License, which permits unrestricted use, distribution, and reproduction in any medium, provided the original work is properly cited.

\begin{abstract}
Background. The efficacy of acupuncture in dry eye syndrome patients remains controversial. Methods. Pubmed, Ovid, Cochrane libraries, CNKI, Wanfang, and CQVIP databases were electronically searched until October 1, 2014. Outcomes including tear break-up time (BUT), Schirmer I test (SIT), and cornea fluorescein staining (CFS) were analyzed. A meta-analysis was performed using both fixed- and random-effects models based on heterogeneity across studies. Results. Seven studies were included in this study; 198 and 185 patients were randomly treated with acupuncture and artificial tears, respectively. The overall BUT of patients in acupuncture group was significantly longer than that of the artificial tears group after treatment $(P<0.00001)$. The SIT was significantly higher in the acupuncture group than that in the artificial tears group after treatment $(P=0.001)$. The CFS of patients in acupuncture group was significantly improved compared to that in artificial group $(P<0.0001)$. Conclusions. Acupuncture therapy is effective for the dry eye patients, partly better than artificial tear treatment.
\end{abstract}

\section{Introduction}

Dry eye syndrome, a worldwide health burden, is a common ocular disorder of the tear film caused by decreased tear production or increased evaporation. Dry eye is highly prevalent, approximately affecting $14 \%$ to $33 \%$ of the adult population worldwide [1]. According to previously published literature, the overall prevalence of dry eye syndrome is estimated to be $5 \%$ to $35 \%$ in various populations [2]. The disease causes significant loss of productivity at work as there is no effective therapy [3].

Currently, in many countries, the recommended therapy for dry eye syndrome is artificial tears as lubricants or supplements for tear deficiency [4]. However, relief from artificial tears lasts only 30-40 minutes, and frequent applications are necessary, so a more effective method is needed for this disease. Acupuncture treatment which has been used for thousands of years in China and has its effectiveness has been provided for some diseases. The efficacy of acupuncture for dry eye syndrome has also been explored; recent studies $[5,6]$ have suggested that acupuncture should be helpful for dry eye syndrome, but other studies $[7,8]$ failed to reach a consensus as to which acupuncture treatment is the most effective. Some systematic reviews [9-11], which include papers that used thunder-fire miraculous moxa in treatment group, concluded proved limited evidence that acupuncture has effectiveness than artificial tears. However, the total number of randomized controlled trials (RCTs), the data accuracy, and the methodological quality in these reviews were too low to draw firm conclusions [9].

According to the concerns mentioned above, to evaluate the efficacy of acupuncture versus artificial tears for dry eye syndrome, we performed a meta-analysis based on more strict inclusion and exclusion criteria for high-quality RCTs. With more accurate methodology, we also compared the overall baseline features, including BUT and SIT, before and after treatment to avoid potential biases.

\section{Materials and Methods}

2.1. Search Strategy. We searched Pubmed, Ovid, Cochrane libraries, CNKI, Wanfang, and CQVIP databases until October 1 , 2014. The following medical subject headings were 
used: "dry eye syndrome," "dry eye," "xerophthalmia," "keratoconjunctivitis sicca," "Gan Yan Zheng," "Gan Yan," "Zhen Ci," "Zhen Jiu," and "acupuncture." Electronic searches were supplemented with manual searches of reference lists of all retrieved review articles, primary studies, and abstracts from meetings to identify other studies not found in the electronic searches. Literature was searched by two authors (L. Yang and Z. Yang) independently.

2.2. Study Selection. Two authors independently selected trials and discussed with each other when inconsistencies were found. Articles that meet the following criteria were included: (1) regarding study types, they should be of randomized controlled trials; (2) regarding participants, they should be dry eye syndrome patients randomly divided; (3) there should be interventions, acupuncture, and artificial tears; (4) regarding outcome measures, studies that used one or more of the following measurements were eligible: BUT, SIT, CFS, and visual analogue scale (VAS); and (5) full texts should be available.

Studies with the following situations were excluded: (1) studies that included other treatments in acupuncture group or control group (e.g., thunder-fire miraculous moxa in acupuncture group); (2) participants with Sjögren's Syndrome; (3) acupuncture combined with other treatments including warming-promotion needling, moxibustion, or Chinese herbal.

2.3. Quality Assessment. The methodological qualities of the included RCTs were assessed according to Cochrane Collaboration's tool described in Handbook version 5.1.0 [12]. The items related to quality assessment included random sequence generation, allocation concealment, blinding of participants and personnel, blinding of outcome assessment, incomplete outcome data, selective reporting, and other biases [12]. Two authors (Z. Yang and L. Yang) assessed the quality independently and inconsistency was discussed with a third review author (H. Song) who acted as an arbiter.

2.4. Data Extraction. Two researchers read the full texts independently and extracted the following contents: publication data, study design, sample size, patient characteristics, treatment protocol (points used, needle reaction time, number of treatment sessions, and frequency of treatment), and outcome measures (BUT, SIT, CFS, and VAS). The authors were contacted by e-mail for additional information if data were unavailable.

2.5. Definitions. Tear film BUT and SIT are considered as the primary outcomes. The BUT is performed as follows: sodium fluorescein $(2.5 \%)$ was applied to both eyes and the interval between the blink of eyes and the first appearance of a dry spot or disruption in the tear film was measured. If BUT is below $10 \mathrm{sec}$, it suggests at least a moderate severity of dry eyes [13-15]. The SIT is a diagnostic method to measure the basic quantity of tear secretion. After application of local anaesthesia, Schirmer test paper (Color Bar, Eagle Vision, USA) was placed in the lateral third of the lower eyelids for 5 minutes with closed eyes. If the SIT result is below $10 \mathrm{~mm} / 5 \mathrm{~min}$, it also suggests at least a moderate severity of dry eyes $[14,15]$.

As second outcomes, CFS is based on Van Bijsterveld scoring system that intensity of stain is scored in two exposed conjunctival zones (nasal and temporal) and cornea. Score of 0 to 3 is given for each zone where 0 is for no stain, +1 for separate spot, +2 for many separate spots, and +3 for confluent spots [16]. A $100 \mathrm{~mm}$ VAS for self-assessment of ocular discomfort was reported by participants. Ocular symptoms related to dry eye (e.g., ocular itching, foreign body sensation, burning, pain and dryness, blurred vision, sensation of photophobia, ocular redness, and sensations of tearing) were quantified and summarised in a standard $100 \mathrm{~mm}$ VAS scale.

2.6. Statistical Methods. Data were processed in accordance with the Cochrane Handbook [12]. Intervention effects were expressed as ORs and associated with $95 \%$ confidence intervals (CIs) for dichotomous data and mean differences and 95\% CIs for continuous data. Subgroup continuous data of each study were combined using the following formula [17]:

$$
\begin{aligned}
\mathrm{SD}= & \left(\left(N_{1}-1\right) \mathrm{SD}_{1}^{2}+\left(N_{2}-1\right) \mathrm{SD}_{2}^{2}\right. \\
& \left.+\frac{N_{1} N_{2}}{N_{1}+N_{2}}\left(M_{1}^{2}+M_{2}^{2}-2 M_{1} M_{2}\right)\right) \\
& \left.\cdot\left(N_{1}+N_{2}-1\right)^{-1}\right)^{1 / 2},
\end{aligned}
$$

where SD is the standard deviation, $N$ is the sample size, and $M$ is the mean.

Heterogeneity across studies was informally assessed by visually inspecting forest plots and formally estimated by Cochran's $Q$ test in which chi-square distribution is used to make inferences regarding the null hypothesis of homogeneity (considered significant at $P<0.10$ ). A rough guide to our interpretation of $I^{2}$ was listed as follows:

(i) $0 \%$ to $40 \%$ shows that heterogeneity may not be important,

(ii) $30 \%$ to $60 \%$ corresponds to moderate heterogeneity,

(iii) $50 \%$ to $90 \%$ exhibits substantial heterogeneity,

(iv) $75 \%$ to $100 \%$ indicates considerable heterogeneity [12, $18]$.

If the eligibility of some studies in the meta-analysis was uncertain because of missing information, a sensitivity analysis was performed by conducting the meta-analysis twice: in the first meta-analysis, all of the studies were included; in the second meta-analysis, only those that were definitely eligible were included. A fixed-effects model was used initially for our meta-analyses; a random-effects model was then used in the presence of heterogeneity. Description analysis was performed when quantitative data could not be pooled. Review Manager version 5.1 software was used for data analysis. 


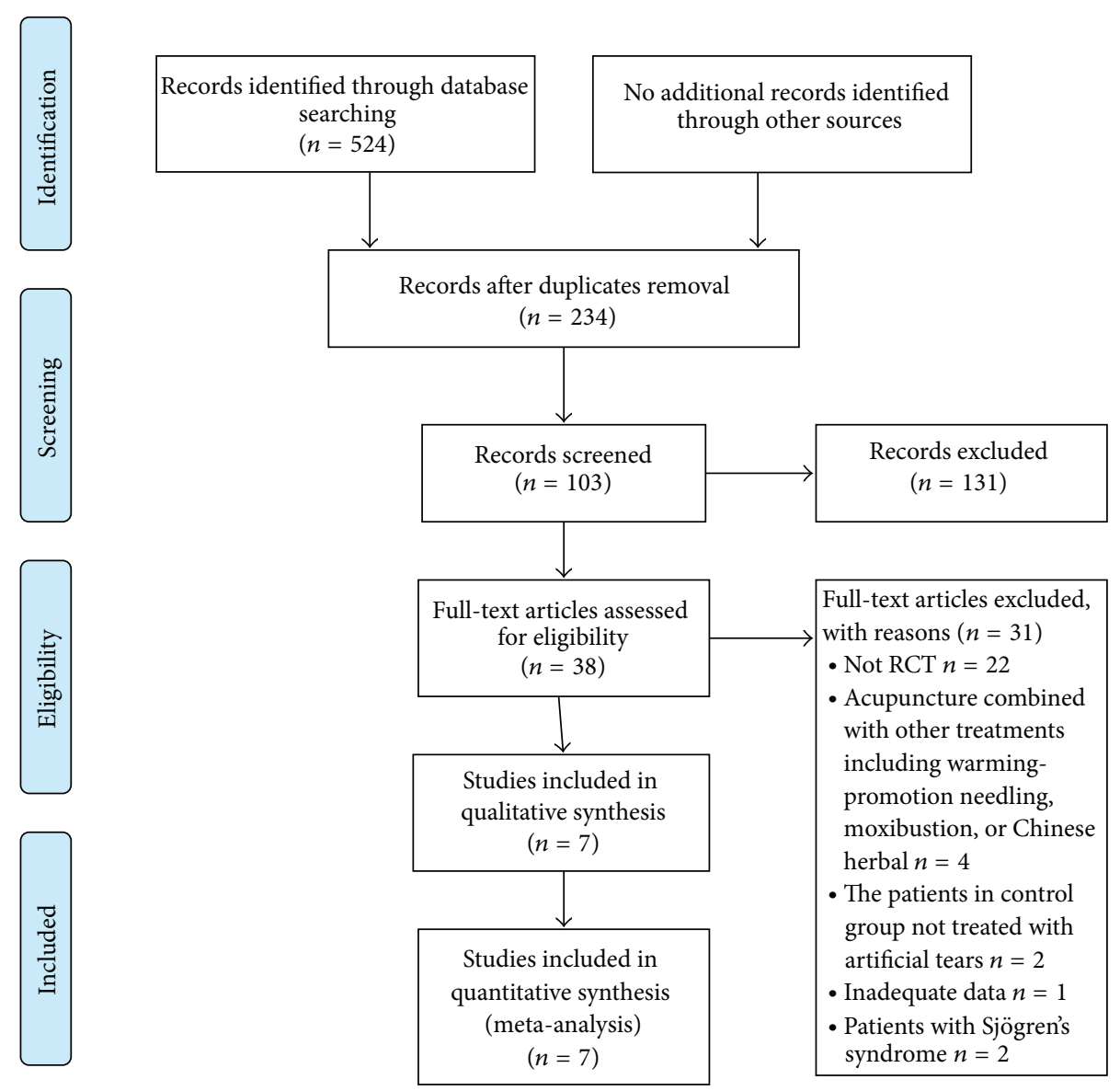

FIgURE 1: Flow diagram of the study selection process.

\section{Results}

3.1. Study and Patient Characteristics. As shown in Figure 1, a total of 103 abstracts were reviewed; among these articles, 38 were retrieved, including 11 RCTs $[5,19-28]$ that are closely related to the current subject. However, three studies [19-21] were excluded because warming-promotion needling technique was used in acupuncture group and one was [22] excluded as Chinese herbal was added in treatment group. Finally, 7 trials [5, 23-28] met our inclusion criteria (Table 1). In total, 198 and 185 patients were randomly treated with acupuncture and artificial tears, respectively. The baseline characteristics of each study included in this meta-analysis are described in Table 2.

3.2. Methodological Quality Assessment. All studies included in this meta-analysis were described as randomized. Four studies [23-25, 27] did not report the method of randomization, but randomization was adequate in other studies [5, $26,28]$. Among these studies, two studies were randomized by a table of random numbers $[5,26]$ and one was randomized through computerised block-randomisation with the SAS package [28]. All studies were performed in common population, which were considered low risk in selection bias. And opaque assignment envelopes with consecutive numbers for each centre were used for allocation concealment in the study reported by Kim et al. [28]. Three reports were blinddesigned [5, 23, 27]. In the study by Tseng et al. [27], apart from the acupuncturist, none of the medical professionals or technicians involved in the study knew to which group a subject had been assigned, while Shi and Miao' report [5] blinded to researchers both sample collection and examination. The outcome assessment of the study by Kim et al. [28] was analyzed by separate statistician, which was considered low risk of detection bias. More than $20 \%$ of participants were lost to follow-up in the study reported by Grönlund et al. [24] and the follow-up data was nonavailable in the study by Wang et al. [26], which were both considered high risk in attrition bias. Also, some outcomes were not reported in results section in Grönlund et al's study [24], which was high risk in reporting bias. Other potential biases were unclear in these trials (Figure 2). As shown in Figure 2, most of the studies included in this meta-analysis achieved low risk of biases of quality assessment items according to Cochrane Handbook for Systematic Reviews of Interventions [12]. Two studies $[24,26]$ had high risk of incomplete outcome data and/or selective reporting. Other biases are unclear.

3.3. BUT. Meta-analysis of five studies [5, 25-28] demonstrated that heterogeneity was not significant among 







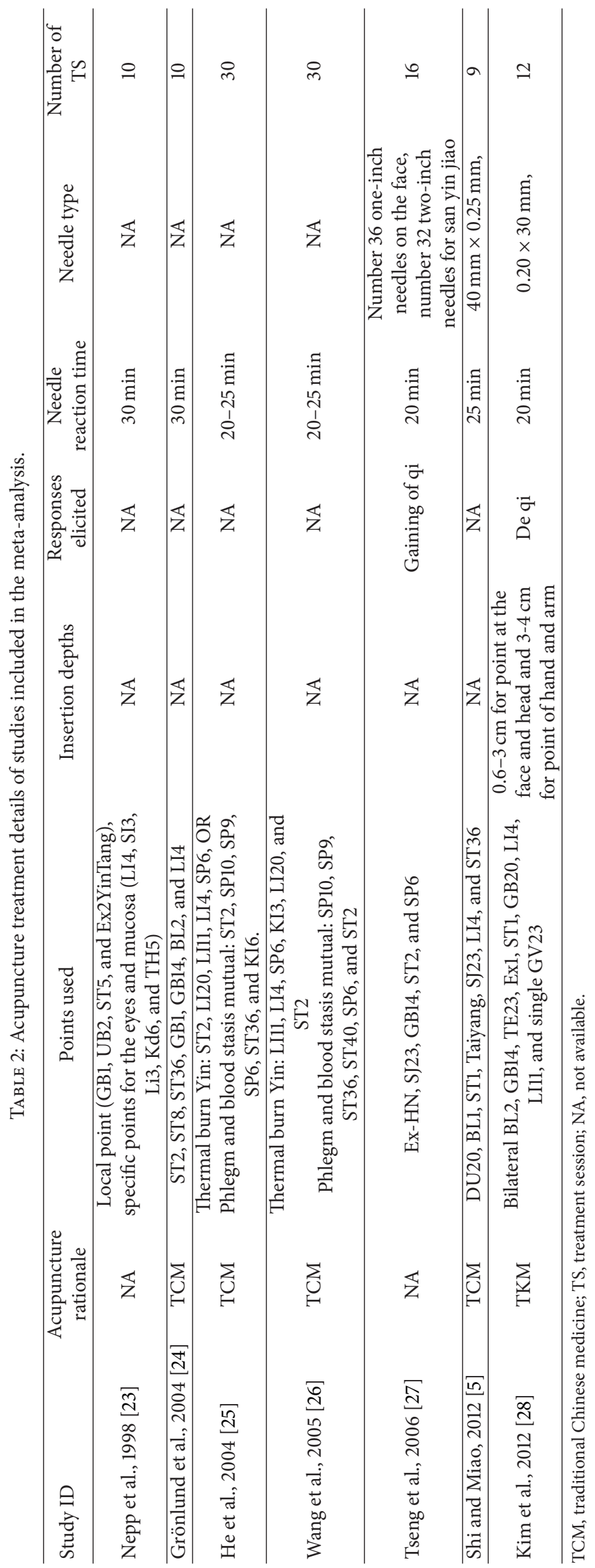




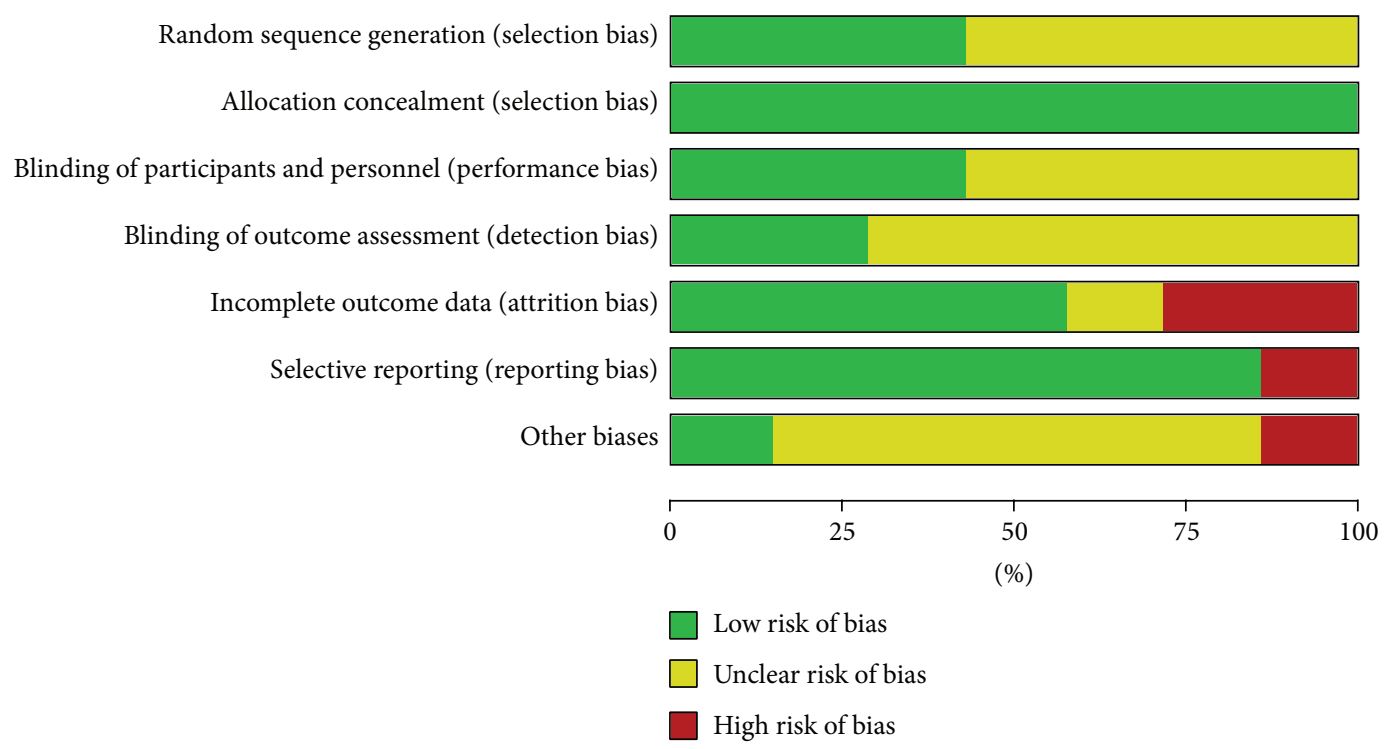

(a)

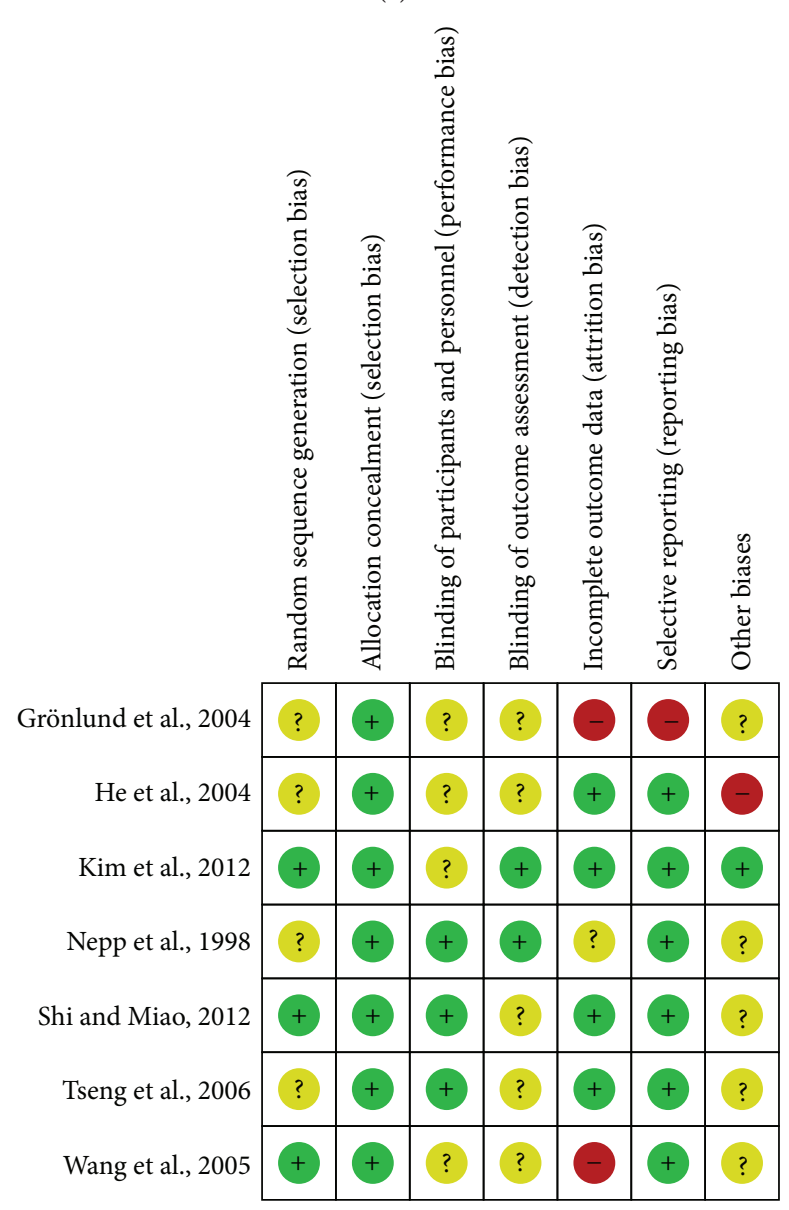

(b)

FIGURE 2: Risk of bias assessment. (a) Risk of bias graph: review authors' judgements about each risk of bias item presented as percentages across all included studies. (b) Risk of bias summary: review authors' judgements about each risk of bias item for each included study. 


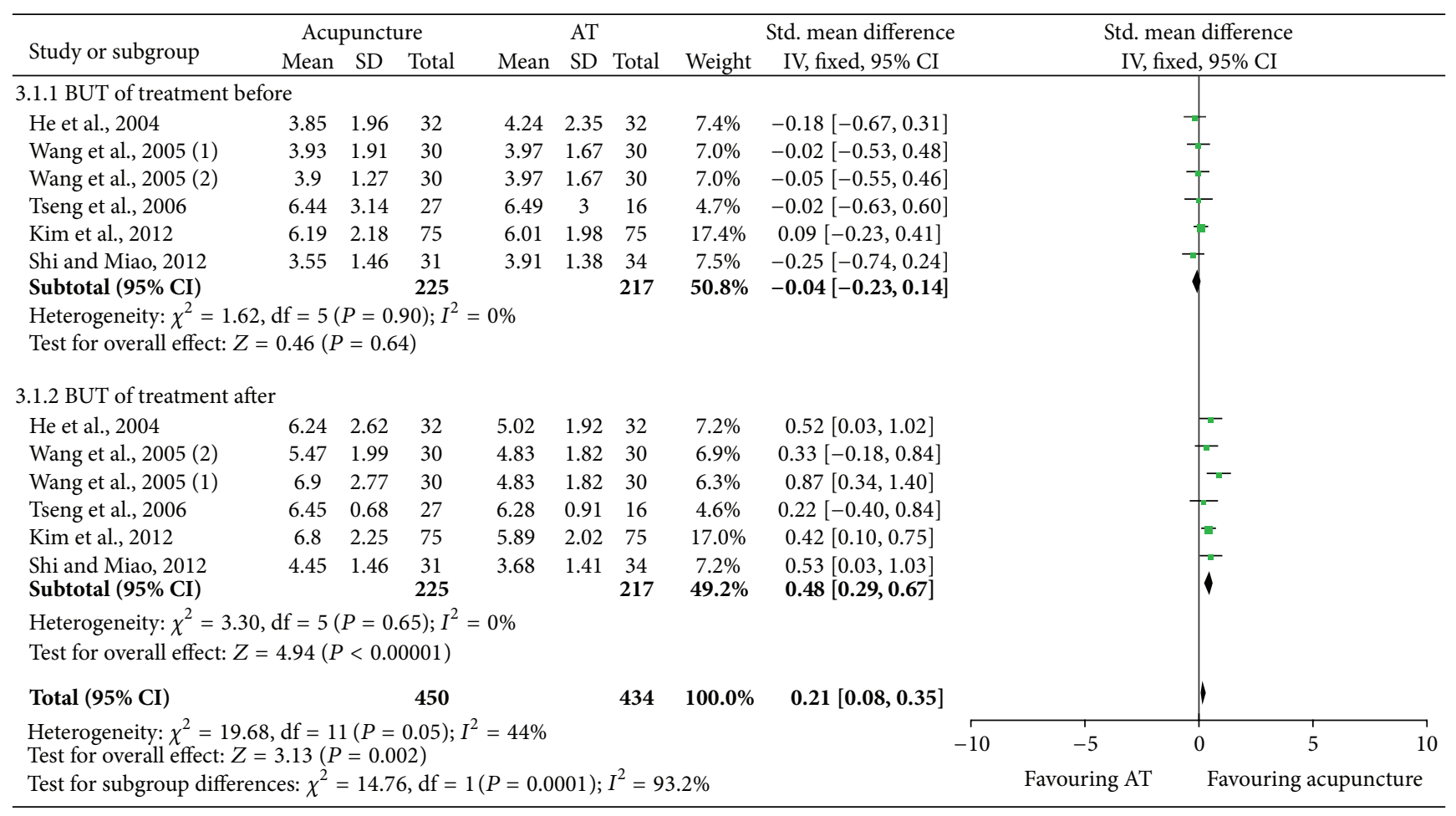

FIGURE 3: BUT comparison between acupuncture and artificial tears (AT) treatment for dry eye syndrome.

the included studies when comparing overall BUT in acupuncture and artificial tears groups both before and after treatment $\left(P=0.90, I^{2}=0 \%\right.$ and $P=0.65, I^{2}=0 \%$, resp., Figure 3). The overall pretreatment BUT of the acupuncture group and artificial tears group showed no significant difference $(P=0.64$, Figure 3$)$. And the overall BUT of posttreatment was significantly longer in the acupuncture group than artificial group $(P<0.00001$, Figure 3$)$. Similarly, the study of Nepp et al. [23] also revealed that acupuncture was more effective than artificial tears in BUT. In contrast, Grönlund et al. [24] concluded that acupuncture was no more effective than receiving a tear substitute in increasing tear quality as indicated by the BUT. Based on the results above, we assumed that acupuncture could prolong the BUT in dry eye syndrome patients.

3.4. SIT. Heterogeneity was not significant among the included studies before treatment $\left(P=0.19, I^{2}=32 \%\right.$, Figure 4). However, the heterogeneity was significant when comparing posttreatment SIT in acupuncture and artificial tears groups $\left(P<0.00001, I^{2}=94 \%\right.$, Figure 4). Thus, random model of meta-analysis showed that acupuncture could significantly improve SIT compared to artificial tears ( $P=0.001$, Figure 4$)$. Similarly, the study of Yang et al. [22] revealed that acupuncture was more effective than artificial tears in SIT. The study by Grönlund et al. [24] did not find any overall difference of SIT improvement between acupuncture and artificial tears group. However when the eyes were analyzed separately, there were a significant increase of SIT in the left eyes over time $(P=0.0131)$. Thus, patients with dry eye syndrome should beneficial from acupuncture in SIT compared to artificial tears.

3.5. CFS. Three studies [24-26] presented CFS outcome and two $[25,26]$ were included in our meta-analysis. Considering that no significant heterogeneity was found among the included studies $[25,26]$ when comparing CFS of posttreatment between acupuncture and artificial tears $(P=0.42$, $I^{2}=0 \%$, Figure 5), our analysis showed that the CFS of patients in acupuncture group had a significant improvement than that of artificial tears group $(P<0.0001$, Figure 5). Since little data is available, further analysis should be performed to evaluate the efficacy of acupuncture in CFS improvement in this population.

3.6. VAS. Grönlund et al. [24] revealed that, according to the VAS recordings, six patients in the acupuncture treatment group felt better after finishing the treatment and no one felt worse, and in the control group no patient felt better and two patients felt worse over the same period of time $(P=0.036)$, but the positive effect had declined after completing the acupuncture treatment range of three to eight months, and there was no statistically significant difference between the two groups. In the study of Tseng et al. [27], the acupuncture group showed greater improvements in VAS values versus control group after eight-week treatment $(P<$ 0.01). However, another study by Kim et al. [28] showed that statistically significant improvements did not appear at two weeks in the acupuncture group and in the artificial tears group $(P=0.359)$ or four weeks of treatment $(P=0.530)$, but 


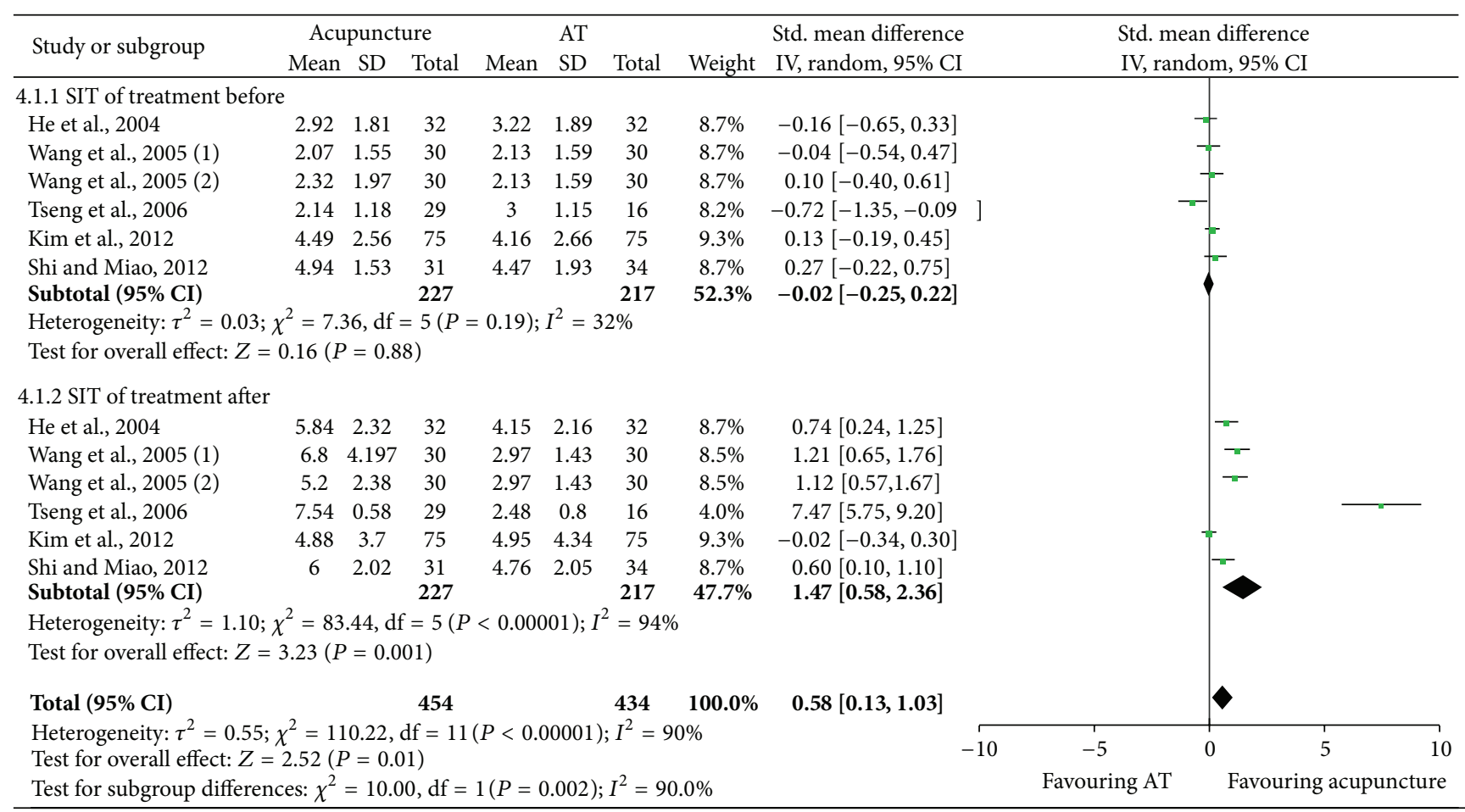

FIGURE 4: SIT comparison between acupuncture and artificial tears (AT) treatment for dry eye syndrome.

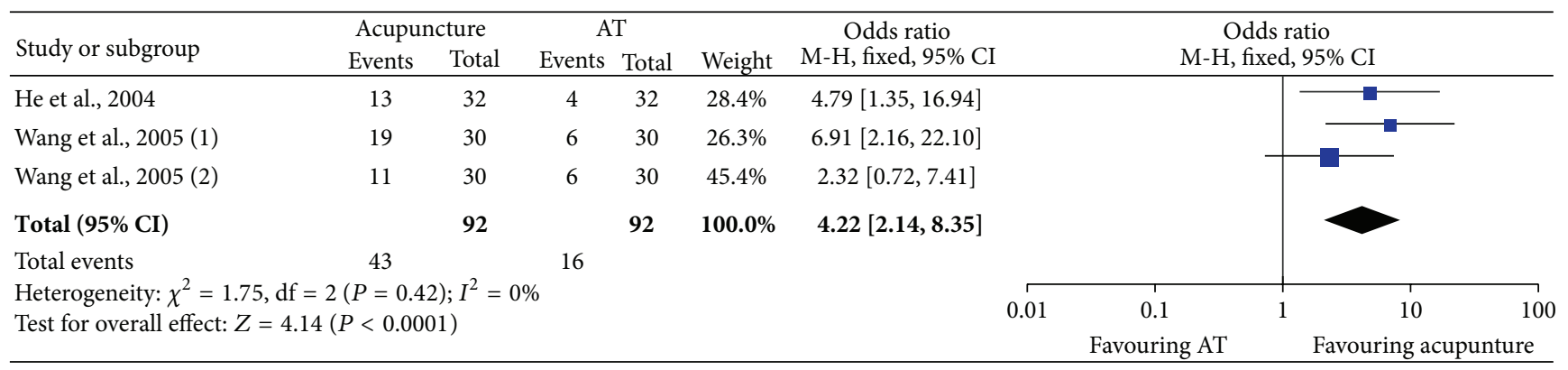

FIGURE 5: CFS comparison between acupuncture and artificial tears (AT) treatment for dry eye syndrome.

significant changes were reported in the acupuncture group at eight weeks after acupuncture treatment compared with control group $(P=0.018)$. In this condition, no confirmed statement of VAS for acupuncture therapy could be presented in this meta-analysis.

3.7. Symptoms of Eye Discomfort. Kim et al. showed that more participants in the acupuncture group experienced general improvements of symptoms than that in the artificial tears group, but there were no significant differences $(P=0.06)$; however, a statistically significant difference was reported between the two groups in the general improvements assessed by physicians $(P=0.001)$. Other two studies $[24,27]$ showed that symptom of eye discomfort in the acupuncture group had improved, but no statistically significant with artificial tears group. Further researches are needed for quality-of-life evaluation in dry eye syndrome patients receiving acupuncture.
3.8. Adverse Event. Only two studies $[24,28]$ included in our meta-analysis reported adverse events. Grönlund et al. [24] reported no adverse effects of the acupuncture treatment per se were noted. In the study by Kim et al. [28], three cases of hematoma were reported in the acupuncture treatment group; 1 patient had moderate severity, and the others had mild severity. Among them, one participant declined further acupuncture treatment because of the hematoma and pain. In the other two cases, the hematomas completely disappeared in several weeks. Adverse events related to artificial tear usage were not reported.

\section{Discussion}

The therapy of dry eye traditionally involved hydrating and lubricating the ocular surface, which may provide temporary improvement in symptoms of irritation and blurred vision, 
but did not address the inflammation that is the underlying cause of dry eye [29]. With acupuncture being one of the oldest interventions, its mechanism in treating dry eye is unknown. Most studies $[30,31]$ thought that the therapeutic effect of acupuncture to dry eye syndrome was from the nervous, hormonal, and immunological systems which were closely tied to etiology of dry eye syndrome. As an active treatment for dry eye syndrome, artificial tears are generally recommended as a first-line therapy drug [32]. Considering this perspective, comparing acupuncture and artificial tears is a basic step for establishing the effectiveness of acupuncture treatment for dry eyes.

The previous meta-analyses [9-11] concluded that there were limited evidence to prove the effectiveness of acupuncture for treating dry eye due to having a large sample size and low methodological quality. Therefore, a more clear evaluation on the acupuncture and artificial tears treatments in dry eye syndromes is essential. In the present meta-analysis, we restricted our high-quality RCTs to acupuncture therapy on dry eye syndrome with non-Sjögren's Syndrome. We excluded studies that acupuncture treatment group combined with other treatments including warming-promotion needling, moxibustion, or Chinese herbal in order to reduce potential biases. In addition, we compared pre- and posttreatment indexes of the main outcomes including BUT and SIT in our analysis, which might contribute to more objective conclusions.

It is often incorrectly assumed that symptoms of dry eye are the main feature of this disease [33]. Symptom questionnaires allow for rapid and efficient collection of relevant information and can facilitate diagnosis of ocular surface disorders [34]. Questionnaires and dry eye index scores can be useful to detect the presence of dry eye and to evaluate the effect of therapeutic treatment. Three studies [24, 27, 28] of our analysis showed that the symptoms of eye discomfort had no statistically significant improvements between acupuncture group and artificial tears group. However, Kim et al. [28] showed that there were statistical significant improvements in symptoms compared to the artificial tear group. Our results indicated that high-quality trials with a large sample size are needed to estimate the efficacy of the acupuncture in improved symptoms of dry eye. Our meta-analysis suggests that the acupuncture group shows greater improvements in VAS values versus control group after treatment. The study by Kim et al. [28] also showed that there was no significant improvement at four weeks when the acupuncture group had just finished the treatment when compared to the artificial tear group, but significant changes were reported in the acupuncture group at four weeks after acupuncture treatment was compared with control group. These findings might be associated with the cumulative effect of acupuncture. Our analysis concluded that acupuncture has an effect in improving the VAS scores for patients of dry eye syndrome.

Currently available diagnostic tests and external examinations are also indispensable for every practitioner in order to reach the decision on the most suitable treatment [35]. Objective tests for dry eye can be divided into tests that examine the tears and those that examine the integrity of the ocular surface. The former can further be subdivided into tests that investigate the quantity, quality, or functional properties of tears [33].

The BUT is the most common test for determining tear film quality in use today [36]. Our results indicated that acupuncture has an effect in improving the BUT of dry eye syndrome patients. On the other hand, Schirmer I test is the most widely used technique to evaluate tear quantity [37]. Our analysis concluded that acupuncture has an effect in improving the SIT for patients of dry eye syndrome.

Cornea fluorescein staining is useful in assessing dry eye where its application can determine the integrity of the corneal and conjunctival epithelium [14, 37]. Our metaanalysis suggests that the CFS of acupuncture group had a more significant improvement than artificial group $(P<$ 0.0001 ). Since small samples and few studies were included in this analysis, high-quality trials with a large sample size are needed to estimate the efficacy of the acupuncture in improved CFS of dry eye syndrome.

One study showed that, according to the VAS recordings [24], six patients in the acupuncture treatment group felt better after finishing the treatment and no one felt worse; in the artificial group no patient felt better and two patients felt worse at the same time $(P=0.036)$; however, no statistical significance could be found in the total number of subjective symptoms, dosage frequency, or, as indicated by the dry eye tests, tear quality, tear secretion, and ocular surface disease in the study. The author concluded that acupuncture has subjective beneficial effects in patients with dry eye syndrome.

In our analysis, we included participants with nonSjögren's Syndrome and excluded combined treatments that might increase the positive results of acupuncture. More importantly, we compared pre- and posttreatment indexes of the main outcomes including BUT and SIT in our analysis, which might contribute to more objective conclusions. Current evidence suggests that acupuncture has superior efficacy in BUT, SIT, CFS, and VAS than artificial tears for dry eye syndrome. Acupuncture therapy is effective for the dry eye patients, partly better than artificial tear treatment. However, our review still had small sizes, relative low quality trials, and short treatment duration. High-quality randomized controlled trials with large samples, long treatment duration, double-blind design, and objective outcomes need to be conducted to evaluate the efficacy and tolerability of acupuncture in this population for a confirmed conclusion in the future. On the other hand, different acupunctulre points and methods, sustained time of acupuncture efficacy, and the correlation of acupuncture effect with treatment duration are also to be conducted.

\section{Conflict of Interests}

The authors declare that they have no competing interests.

\section{Authors' Contribution}

Lei Yang and Zongguo Yang equally contributed to this paper. 


\section{References}

[1] S. C. Pflugfelder, "Prevalence, burden, and pharmacoeconomics of dry eye disease," The American Journal of Managed Care, vol. 14, no. 3, pp. S102-S106, 2008.

[2] J. A. Smith, J. Albenz, C. Begley et al., "The epidemiology of dry eye disease: report of the epidemiology subcommittee of the international Dry Eye WorkShop (2007)," The Ocular Surface, vol. 5, no. 2, pp. 93-107, 2007.

[3] M. A. Lemp, C. Baudouin, J. Baum et al., "The definition and classification of dry eye disease: report of the definition and classification subcommittee of the international Dry Eye WorkShop (2007)," Ocular Surface, vol. 5, no. 2, pp. 75-92, 2007.

[4] M. A. Lemp, "Management of dry eye disease," The American Journal of Managed Care, vol. 14, supplement 3, pp. S88-S101, 2008.

[5] J. L. Shi and W. H. Miao, "Effects of acupuncture on lactoferrin content in tears and tear secretion in patients suffering from dry eyes: a randomized controlled trial," Journal of Chinese Integrative Medicine, vol. 10, no. 9, pp. 1003-1008, 2012 (Chinese).

[6] J.-H. Jeon, M.-S. Shin, M. S. Lee et al., "Acupuncture reduces symptoms of dry eye syndrome: a preliminary observational study," The Journal of Alternative and Complementary Medicine, vol. 16, no. 12, pp. 1291-1294, 2010.

[7] M.-S. Shin, J.-I. Kim, M. S. Lee et al., "Acupuncture for treating dry eye: a randomized placebo-controlled trial," Acta Ophthalmologica, vol. 88, no. 8, pp. e328-e333, 2010.

[8] T. List, T. Lundeberg, I. Lundström, F. Lindström, and N. Ravald, "The effect of acupuncture in the treatment of patients with primary Sjögren's syndrome: a controlled study," Acta Odontologica Scandinavica, vol. 56, no. 2, pp. 95-99, 1998.

[9] M. S. Lee, B. C. Shin, T. Y. Choi, and E. Ernst, "Acupuncture for treating dry eye: a systematic review," Acta Ophthalmologica, vol. 89, no. 2, pp. 101-106, 2011.

[10] J. Su, X. Q. Liu, W. H. Miao et al., "Acupuncture therapy in treating keratoconjunctivitis sicca: a systematic review," Journal of Traditional Chinese Ophthalmology, vol. 24, no. 1, pp. 40-46, 2014.

[11] J. Ba, Y. Wu, Y. Li, D. Xu, W. Zhu, and J. Yu, "Updated metaanalysis of acupuncture for treating dry eye," Medical Acupuncture, vol. 25, no. 5, pp. 317-327, 2013.

[12] J. P. T. Higgins and S. Green, Eds., Cochrane Handbook for Systematic Reviews of Interventions Version 5.1.0, The Cochrane Collaboration, 2011, http://www.cochrane-handbook.org/.

[13] K. Gumus and D. H. Cavanagh, "The role of inflammation and antiinflammation therapies in keratoconjunctivitis sicca," Clinical Ophthalmology, vol. 3, no. 1, pp. 57-67, 2009.

[14] P. D. O'Brien and L. M. T. Collum, "Dry eye: diagnosis and current treatment strategies," Current Allergy and Asthma Reports, vol. 4, no. 4, pp. 314-319, 2004.

[15] K. L. Krenzer, M. R. Dana, M. D. Ullman et al., "Effect of androgen deficiency on the human meibomian gland and ocular surface," The Journal of Clinical Endocrinology \& Metabolism, vol. 85 , no. 12 , pp. 4874-4882, 2000.

[16] O. P. van Bijsterveld, "Diagnostic tests in the Sicca syndrome," Archives of Ophthalmology, vol. 82, no. 1, pp. 10-14, 1969.

[17] J. Luo and W. D. Leng, Eds., Theory \& Practice of Systematic Review/Meta-Analysis, Military Medical Science Press, 2013.

[18] J. P. T. Higgins and S. G. Thompson, "Quantifying heterogeneity in a meta-analysis," Statistics in Medicine, vol. 21, no. 11, pp. 15391558, 2002.
[19] X.-H. Xu and X.-L. Fang, "Efficacy observation of xerophthalmia treated with acupuncture of warming-promotion needling technique," Zhongguo Zhen Jiu, vol. 32, no. 3, pp. 233236, 2012 (Chinese).

[20] L.-X. Wei, W. Yang, H.-C. Wang, O. Zhang, R.-Q. Ding, and Z.H. Liu, "Efficacy assessment of acupuncture and moxibustion on tear secretion in xerophthalmia," Zhongguo Zhen Jiu, vol. 30, no. 9, pp. 709-712, 2010 (Chinese).

[21] Y. Zhang and W. Yang, "Effects of acupuncture and moxibustion on tear-film of the patients with xerophthalmia," Journal of Traditional Chinese Medicine, vol. 27, no. 4, pp. 258-260, 2007.

[22] W. Yang, Y. C. Zhang, D. Liu, H. Sun, and L. Q. Chen, "Clinical observation on the method for nourishing blood and moisturizing eye on xerophthalmia," Zhongguo Zhen Jiu, vol. 26, no. 8, pp. 571-573, 2006 (Chinese).

[23] J. Nepp, A. Wedrich, J. Akramian et al., "Dry eye treatment with acupuncture-a prospective, randomized, double-masked study," Advances in Experimental Medicine and Biology, vol. 438, pp. 1011-1016, 1998.

[24] M. A. Grönlund, U. Stenevi, and T. Lundeberg, "Acupuncture treatment in patients with keratoconjunctivitis sicca: a pilot study," Acta Ophthalmologica Scandinavica, vol. 82, no. 3, pp. 283-290, 2004.

[25] H. Q. He, Z. L. Wang, H. L. Hu, and R. Liu, "Effect of acupuncture on lacrimal film of Xeroma patients," Journal of Nanjing TCM University, vol. 20, no. 3, pp. 158-159, 2004 (Chinese).

[26] Z. L. Wang, H. Q. He, D. Huang, and C. G. Shi, "Affect of integral syndrome differentiation acupuncture on the tear film stability in the patient of xerophthalmia," Chinese Acupuncture Moxibustion, vol. 25, no. 7, pp. 460-463, 2005 (Chinese).

[27] K. L. Tseng, H. J. Liu, K. Y. Tso, L. C. Woung, Y. C. Su, and J. G. Lin, "A clinical study of acupuncture and SSP (silver spike point) electro-therapy for dry eye syndrome," The American Journal of Chinese Medicine, vol. 34, no. 2, pp. 197-206, 2006.

[28] T.-H. Kim, J. W. Kang, K. H. Kim et al., "Acupuncture for the treatment of dry eye: a multicenter randomised controlled trial with active comparison intervention (artificial teardrops)," PLoS ONE, vol. 7, no. 5, Article ID e36638, 2012.

[29] A. S. Bhavsar, S. G. Bhavsar, and S. M. Jain, "A review on recent advances in dry eye: pathogenesis and management," Oman Journal of Ophthalmology, vol. 4, no. 2, pp. 50-56, 2011.

[30] B. Esmaeli, J. T. Harvey, and B. Hewlett, "Immunohistochemical evidence for estrogen receptors in meibomian glands," Ophthalmology, vol. 107, no. 1, pp. 180-184, 2000.

[31] M. A. Meneray, T. Y. Fields, and D. J. Bennett, "Gi/Go couple met-enkephalin to inhibition of cholinergic and $\beta$-adrenergic stimulation of lacrimal secretion," Cornea, vol. 19, no. 1, pp. 9298, 2000.

[32] S. C. Pflugfelder, G. Geerling, S. Kinoshita et al., "Management and therapy of dry eye disease: report of the management and therapy subcommittee of the international Dry Eye WorkShop (2007)," Ocular Surface, vol. 5, no. 2, pp. 163-178, 2007.

[33] S. Kaštelan, M. Tomić, J. Salopek-Rabatić, and B. Novak, "Diagnostic procedures and management of dry eye," BioMed Research International, vol. 2013, Article ID 309723, 6 pages, 2013.

[34] R. M. Schiffman, M. D. Christianson, G. Jacobsen, J. D. Hirsch, and B. L. Reis, "Reliability and validity of the ocular surface disease index," Archives of Ophthalmology, vol. 118, no. 5, pp. $615-621,2000$. 
[35] A. Behrens, J. J. Doyle, L. Stern et al., "Dysfunctional tear syndrome: a Delphi approach to treatment recommendations," Cornea, vol. 25, no. 8, pp. 900-907, 2006.

[36] P. Buchholz, C. S. Steeds, L. S. Stern et al., "Utility assessment to measure the impact of dry eye disease," Ocular Surface, vol. 4, no. 3, pp. 155-161, 2006.

[37] W. B. Jackson, "Management of dysfunctional tear syndrome: a Canadian consensus," Canadian Journal of Ophthalmology, vol. 44, no. 4, pp. 385-394, 2009. 


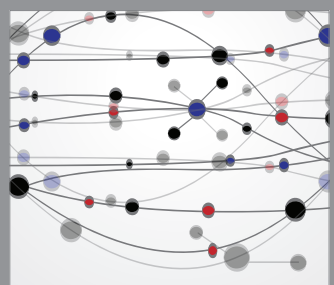

The Scientific World Journal
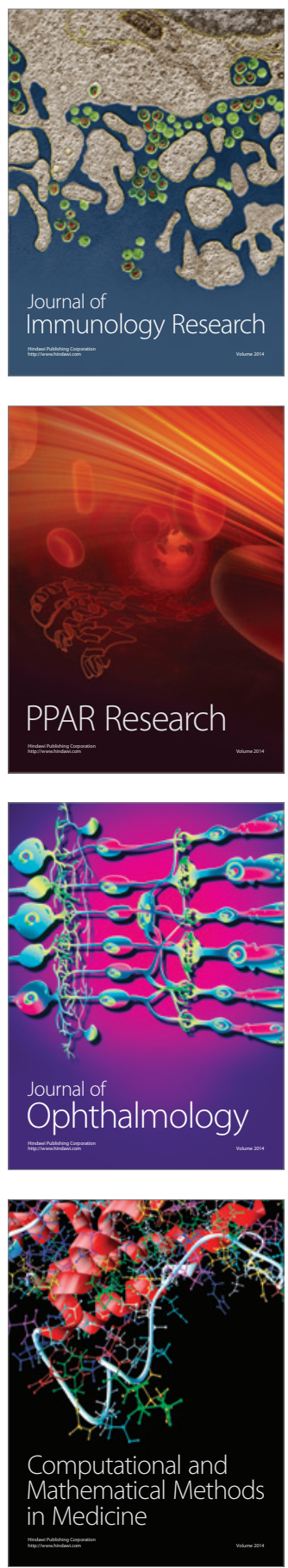

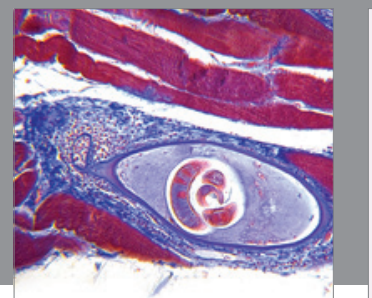

Gastroenterology

Research and Practice
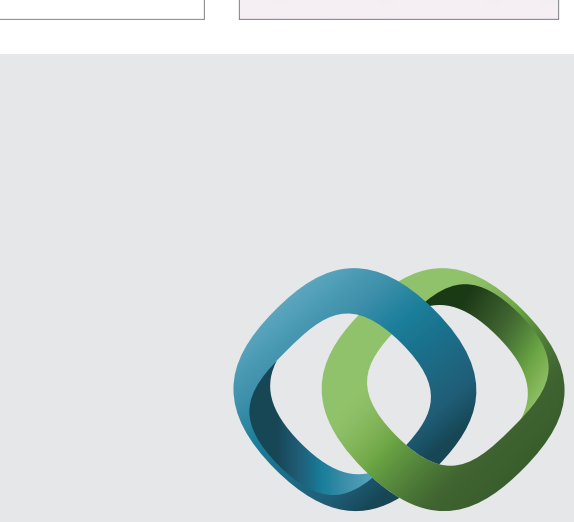

\section{Hindawi}

Submit your manuscripts at

http://www.hindawi.com
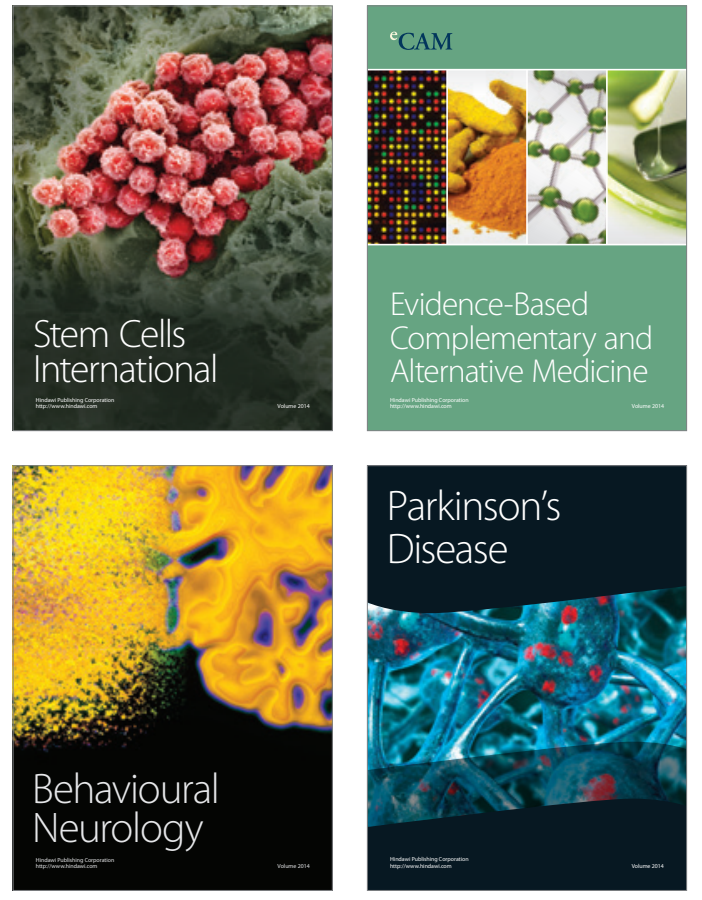
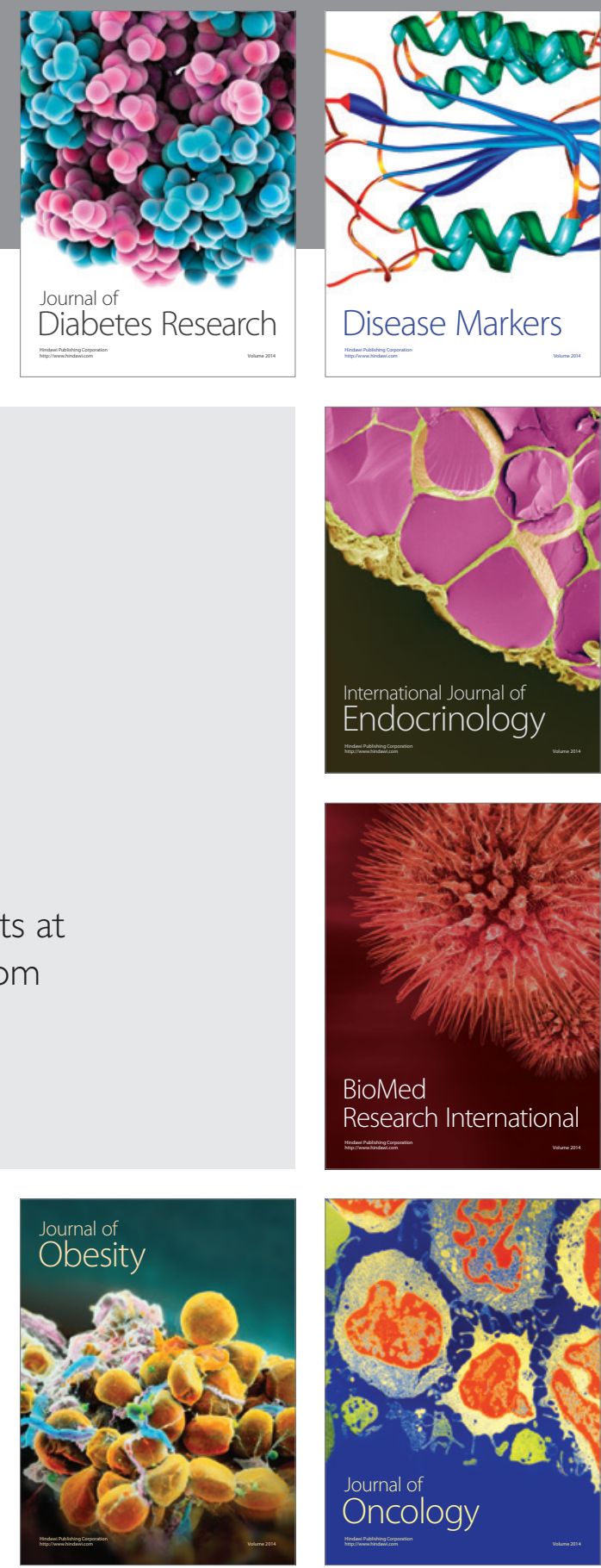

Disease Markers
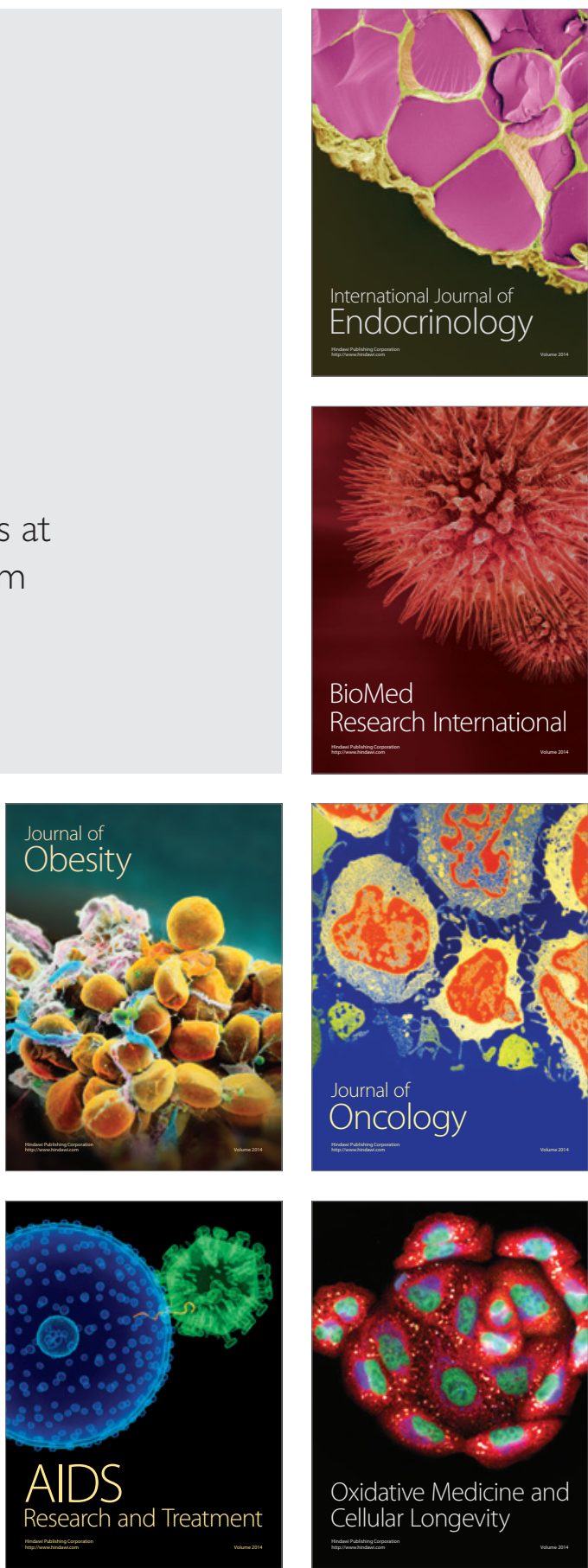